\title{
Teilberufsausübungsgemeinschaften
}

\section{Vorsicht bei Zuweisungen innerhalb der Kooperation}

\author{
Wie dürfen Fach- und Hausärzte ihre Kooperation gestalten, ohne dass \\ sie gegen das Verbot der Zuweisung gegen Entgelt verstoßen? In einem \\ aktuellen Urteil zieht das Oberlandesgericht Karlsruhe eine klare Grenze \\ für Teilberufsausübungsgemeinschaften.
}

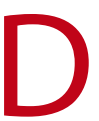
ie Zusammenarbeit im Rahmen einer „Teilberufsausübungsgemeinschaft" (Teil-BAG) ist Fachärzten nach allen Berufsordnungen der Länder grundsätzlich erlaubt. Eine solche Kooperation darf allerdings nicht einer Umgehung des Verbotes der $\mathrm{Zu}$ weisung gegen Entgelt dienen. Eine solche Umgehung liegt - so die Regelung der Berufsordnungen - vor allem dann vor, wenn sich der Beitrag eines Arztes in der Kooperation auf das Erbringen medizinisch-technischer Leistungen auf Veranlassung der übrigen Mitglieder einer Teil-BAG beschränkt. Das hat jetzt das Oberlandesgericht (OLG) Karlsruhe im Fall einer Teil-BAG aus Nordbaden klargestellt (6 U 15/11). Der Kooperation war es nicht gelungen darzulegen, dass die teilnehmenden Radiologen für die Teil-BAG auch Leistungen erbringen, die nicht von Mitgesellschaftern veranlasst wurden. Der Einwand der Gesellschaft, ein Radiologe werde immer auf Überweisung tätig, geht hierbei an der Sache vorbei: Es kommt darauf an, ob der Radiologe auch auf Veranlassung anderer Ärzte, die nicht Mitgesellschafter der Teil-BAG sind, Leistungen erbringt und diese dann als Mitglied der Teilgemeinschaftspraxis, also nicht für seine daneben bestehende Einzel- oder Gemeinschaftspraxis, abrechnet.

Das OLG Karlsruhe stellte ausdrücklich fest, dass eine Teil-BAG unter Einbeziehung von Ärzten der Methodenfächer zulässig ist, wenn ihr Beitrag nicht auf die Erbringung medizinisch-technischer Leistungen auf Veranlassung der übrigen Gesellschafter beschränkt ist, sondern sie darüber hinaus weitere Leistungen erbringen. Nach Auffassung des OLG ist demnach unter dem Begriff der „Leistung“ in diesem Sinne nicht jede beliebige Aktivität, sondern nur eine Tätigkeit, die grundsätzlich gegenüber dem Patienten abrechenbar ist, zu verstehen.

\section{„Berufsordnung" trotz und wegen offener Kooperationsmodelle}

Die Auslegung des OLG soll dazu dienen, nicht jede gemeinsame Befunderörterung als gesonderte Teilleistung oder - gesellschaftsrechtlich - als Beitrag zu deklarieren und hierdurch die Regelung der Berufsordnung zu umgehen. Die streitige Gestaltung wäre also dann zulässig gewesen, wenn die Radiologen ihre Leistungen - es ging vor allem um Knochendichtemessungen - auch dann auf Rechnung der Teilgemeinschaftspraxis erbracht hätten, wenn der veranlassende Arzt nicht Mitgesellschafter der Teilgemeinschaftspraxis ist. Bleibt die Frage, warum die Ärzte dies nicht vereinbart haben? Man kann sich das an einem Beispiel verdeutlichen: Ein Facharzt und ein Laborarzt wollen zusammenarbeiten. Sie könnten sich vollumfänglich zu einer Gemeinschaftspraxis verbinden, der Facharzt will jedoch primär an den Umsätzen „seiner“ Laborproben partizipieren und nicht in die gesamtschuldnerische Haftung für die teuren Laborgeräte eintreten. Der Laborarzt will eigentlich nur den Facharzt als Einsender an sich binden, er will nicht seine gesamten Laborgewinne mit ihm teilen. Weil sich bei dieser Interessenlage das Gemeinschaftliche letztlich auf wirtschaftliche Überlegungen im Bereich der überweisungsgebundenen, also in gewisser Weise steuerbaren Leistungen reduziert, beurteilen die Berufsordnungen der deutschen Ärzte die Zulässigkeit eben auch anders, als wenn sich zwei Ärzte vollumfänglich zur gemeinsamen Berufsausübung zusammenschließen.

Die Berufsordnung diskriminiert keinesfalls Zusammenschlüsse von Ärzten und schafft auch keine ungleichen Rahmenbedingungen im Vergleich zu MVZ. Der Zusammenschluss zur Erbringung aller Leistungen ist Ärzten und MVZ in gleicher Weise erlaubt. Umgekehrt ist es aber sowohl freiberuflichen Ärzten als auch in MVZ angestellten Ärzten untersagt, an einer Teil-BAG mitzuwirken, die sich bei medizinisch-technischen Leistungen darauf beschränkt, diese nur auf Veranlassung anderer Mitgesellschafter und eben nicht auf Überweisung aller Ärzte zu erbringen. Rechtlich muss sich nämlich jede MVZ-Trägergesellschaft, auch wenn für sie die Berufsordnung nicht unmittelbar gilt, das Handeln ihrer angestellten Ärzte zurechnen lassen.

Angesichts der aktuellen rechtlichen Probleme, etwa wegen der Zusammenarbeit mit Kliniken im prä- und poststationären Bereich und der Mitarbeit von niedergelassenen Ärzten bei stationären Operationen dürfte die Teilgemeinschaftspraxis im ärztlichen AllTeil-BAG und im Hinblick auf die rechtlichen Unsicherheiten eher eine untergeordnete Rolle spielen. Die Ärzteschaft braucht ein Berufsrecht, das den - großen - Korridor der zulässigen Kooperationen, aber auch dessen Grenzen definiert. Diese Grenzen müssen eindeutig und für jeden Arzt verständlich sein. Bisher sind sie dies nicht.

\section{Dr. Ingo Pflugmacher}

Fachanwalt für Medizin- und Verwaltungsrecht, Kanzlei Busse \& Miessen, Bonn 